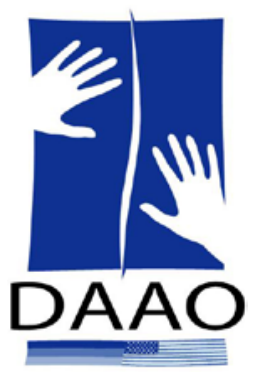

\title{
Deutsch-Amerikanische Akademie für Osteopathie DAAO e.V.
}

\author{
in der Deutschen Gesellschaft für Manuelle Medizin \\ Dr. Karl-Sell-Ärzteseminar Neutrauchburg (MWE) e.V. \\ Riedstraße 5, 88316 Isny-Neutrauchburg, Tel.: 07562-9718-0, Fax: 07562-9718-22 • \\ www.daao.info, info@aerzteseminar-mwe.de
}

Information und Anmeldung:

MWE-Sekretariat, Riedstraße 5, 88316 Isny-Neutrauchburg

Tel.:(07562) 9718-0 - Fax: (07562) 9718-22

E-Mail:info@aerzteseminar-mwe.de - Internet:www.daao.info

\section{GRUNDKURSE}

Einführungskurs

18.06.-20.06.2015 - Isny (E) EAN

03.09.-05.09.2015 - Bad Iburg (D) SG

Counterstrain-I-Kurs

21.06.-23.06.2015 - Isny (E) EAN

06.09.-08.09.2015 - Bad lburg (D) RH

Myofaszial-Release-Kurs

23.07.-25.07.2015 - Isny (D) BK

22.10.-24.10.2015 - Bad lburg (E) EAN

Muskel-Energie-Kurs

26.07.-28.07.2015 - Isny (D) RG

25.10.-27.10.2015 - Bad lburg (E) EAN

Cranio-Sacral-I-Kurs

14.05.-16.05.2015 - Isny (E) ASN

07.11.-09.11.2015 - Isny (E) DA

Visceral-I-Kurs

17.05.-19.05.2015 - Isny (E) ASN

10.11.-12.11.2015 - Isny (E) DA

Lymphatische und ligamentäre Gelenktechniken (LAS)

11.06.-13.06.2015 - Isny (D) OD

22.10.-24.10.2015 - Isny (D) FM

Integrations-Kurs

14.06.-16.06.2015 - Isny (D) SG

25.10.-27.10.2015 - Isny (D) SS

AUFBAUKURSE

Counterstrain-II-Kurs

24.09.-26.09.2015 - Bad lburg (E) ASN

Cranio-Sacral-II-Kurs

14.06.-16.06.2015 - Bad Iburg (D) FM

Visceral-II-Kurs

18.10.-20.10.2015 - Isny (D) SF

Kursleiter - Legende:

ASN = Prof. Dr. Alexander S. Nicholas OD = Dr. med. Oliver Drieschner

$\mathrm{BK}=$ Dr. med. Bärbel Krebs

$\mathrm{CV}=$ Dr. med. Christof Verres

$\mathrm{DA}=$ Dr. med. Donald Allison

$\mathrm{EAN}=$ Dr. med. Evan Nicholas

$\mathrm{FM}=$ Frank Müller

$\mathrm{GS}=$ Dr.med. Gunnar Spohr

$\mathrm{RG}=$ Dr. med. Rainer Galm

$\mathrm{RH}=$ Dr. med. Rosemarie Hofem

$\mathrm{RP}=$ Dr.med. Rolf Pesch

$\mathrm{SB}=$ Dr. med. Saied Behrouz

$\mathrm{SG}=$ Dr. med. Stefan Giesswein

SS $=$ Sabine Sörries
Still-FPR-Techniken

25.06.-27.06.2015 - Isny (D) FM

17.09.-19.09.2015 - Bad Iburg (D) CV

HVLA

15.10.-17.10.2015 - Isny (D) RG

Osteopathische Woche

05.10.-09.10.2015 - Florida (D)

09.11.-13.11.2015 - Florida (D)

Anatomie als Grundlage der Osteopathischen Medizin 11.10.-13.10.2015 - Isny (D) GS

Postgraduateprogramm 2015

POSTGRADUATE KURSE

Kinderosteopathie: BLT

10.09.-12.09.2015 - Frankfurt (E) Kursleiter: Hugh Ettlinger

13.09.-15.09.2015 - Bad lburg (E) Kursleiter: Hugh Ettlinger

Kinderosteopathie: Birthtrauma

24.05.-26.05.2015 - Wien (E) Kursleiter: Prof. Darren Mc Auley

Kinderosteopathie:

Cranio in the Newborn and the small child (toddler)

07.06.-09.06.2015 - Wien (E) Kursleiter: Heather Ferrill

Sportsosteopathy Special 1

A Clinical Approach to the Ligamentous System

12.11.-14.11.2015 - Frankfurt (E) Kursleiter: Jason Hartmann

Sportsosteopathy Special 2

An Integrated Approach to Osteopathy and Prolotherapy

15.11.-17.11.2015 - Frankfurt (E) Kursleiter: Jason Hartmann

Bioenergetics

19.06.-21.06.2015 - Frankfurt (E) Kursleiter: Jan Hendryx

22.06.-24.06.2015 - Bad Iburg (E) Kursleiter: Jan Hendryx

The old masters visceral course

27.08.-29.08.2015 - Wien (E) Kursleiter: Tom Crow

The old masters musculoskeletal course

30.08.-01.09.2015 - Wien (E) Kursleiter: Tom Crow

Tagesrefresher - Osteopathische Diagnostik

14.11.2015 - Köln (D) Kursleiter: Stefan Giesswein

Behandlungsstrategie - das Konzept

der Schlüsselläsion nach Ed Stiles

19.09.2015 - Köln (D) Kursleiter: Stefan Giesswein

Refresherkurs

30.10.-01.11.2015 - Isny (D) Kursleiter: Saied Behrouz

Die Kurse finden in Deutsch (D) bzw. Englisch (E) mit Übersetzungshilfe statt. $U=$ Kursleiter unbekannt.

Es sind weitere Kurstermine geplant.

Kurse in Wien und in Graz, von PCOM -Dozenten gehalten, werden auch von uns anerkannt.

$\mathrm{MF}=$ Dr. med. Michael Fleischhauer 